\section{Fatores associados a hábitos de saúde bucal e utilização de serviços odontológicos entre adolescentes}

\author{
Factors associated with oral health habits and use \\ of dental services by adolescents
}

\author{
${ }_{1}^{1}$ Programa de Pós-graduação \\ em Saúde Coletiva, \\ Universidade Luterana do \\ Brasil, Canoas, Brasil. \\ 2 Programa de Pós-graduação \\ em Odontologia, \\ Universidade Federal do Rio \\ Grande do Sul, Porto Alegre, \\ Brasil. \\ 3 Secretaria Municipal de \\ Educação de Gravataí, \\ Gravataí, Brasil. \\ Correspondência \\ R. S. Davoglio \\ Programa de Pós-graduação \\ em Saúde Coletiva, \\ Universidade Luterana do \\ Brasil. \\ Av. Guadalupe 380, apto. 405, \\ Porto Alegre, RS \\ 91050-250, Brasil. \\ rosanedavoglio@via-rs.net
}

\section{Abstract}

This was a cross-sectional study of 1,170 seventh-grade adolescents from the municipal public school system in Gravataí, Rio Grande do Sul State, Brazil, investigating the association between socio-demographic, psychosocial, and lifestyle factors and oral health habits and use of dental services. Data were analyzed by Cox regression, modified for cross-sectional studies. Females showed higher frequency of brushing, as did adolescents that reported not feeling alone or discriminated. Daily flossing was associated with higher socioeconomic status (SES), use of private dental services, parental understanding, and lack of feeling of loneliness. Frequency of annual dental visits was higher among individuals with higher SES. Preventive dental checkups were more frequent among individuals with higher SES, those who felt understood by their parents, and those who did not habitually eat candy. Oral health habits were associated with family SES and psychosocial factors except for frequency of annual dental visits. As for lifestyle, low candy consumption had a positive impact on reasons for use of dental appointments.

Dental Health Services; Adolescent; Oral Health
Rosane Silvia Davoglio Denise Rangel Ganzo de Castro Aerts 1 Claídes Abegg 2 Silvia Letícia Freddo 1

Lisiane Monteiro 3

\section{Introdução}

A atitude das pessoas acerca de sua saúde, particularmente a saúde bucal, é moldada por suas vivências pessoais. Essas atuarão como determinantes de comportamentos e percepções, fundamentais na adoção de hábitos de saúde bucal e no desenvolvimento de um padrão de comportamento relacionado aos mesmos 1 .

Fatores sócio-demográficos, psicossociais e o estilo de vida adotado pelo indivíduo podem influenciar seus hábitos e comportamentos de saúde em todas as etapas da vida. O adolescente, no entanto, mostra-se mais vulnerável a esses fatores, uma vez que já não é mais beneficiado pelo cuidado e atenção dispensados às crianças nem desfruta da proteção associada à maturidade da vida adulta ${ }^{2}$. Além disso, a adolescência é considerada um período de risco para doenças bucais como cárie, gengivite e doença periodontal 3 .

Os fatores sócio-econômicos, como o nível de escolaridade, e a percepção da necessidade de tratamento 4,5 atuam como elementos diferenciadores no acesso à saúde, pois influenciam a obtenção de conhecimentos e cuidados. Em relação ao sexo, as mulheres são mais adeptas a hábitos adequados de saúde bucal e cuidados preventivos 6 .

As relações sociais podem atuar como determinantes da saúde bucal 7,8 , uma vez que a convivência entre as pessoas favorece comportamentos de monitoramento, incentivando mui- 
tas atividades pessoais que se associam à melhor qualidade de vida, como dietas, exercícios físicos e cuidados com a saúde ${ }^{9}$. Os comportamentos de saúde geral foram associados com riscos cardiovasculares e doenças bucais, o que confirma a suposição de que compartilham um fundo comportamental comum 10 .

O estilo de vida adotado pelas pessoas, como hábitos alimentares, consumo de tabaco, bebidas alcoólicas e drogas, apresenta-se como fator de risco para doenças bucais 11. A adolescência é um período crítico para a experimentação de drogas lícitas e ilícitas. O uso de tabaco e bebida alcoólica está associado a uma variedade de condições orais patológicas em adultos, e esses comportamentos coexistem na adolescência 12, o mesmo ocorrendo com as drogas ${ }^{13}$. No entanto, a adolescência é também a fase na qual o jovem pode adquirir um aprendizado relacionado a atitudes e comportamentos positivos que persistirão no futuro, representando um momento fundamental para a promoção da saúde 14 .

Em função da vulnerabilidade própria do adolescente, a Organização Mundial da Saúde (OMS) 15 preconiza o monitoramento da saúde do escolar por meio da vigilância de fatores de risco comportamentais e de proteção na faixa etária de 13 a 15 anos, sendo essa já implementada em muitos países. Nessa perspectiva, o Ministério da Saúde tem incentivado os municípios a monitorarem a saúde dos escolares, tendo, como grupo prioritário, os estudantes da sétima série (atualmente denominado oitavo ano) do Ensino Fundamental 16. Assim, esta pesquisa teve por objetivo estudar a associação de fatores sócio-demográficos, psicossociais e relacionados ao estilo de vida com hábitos de saúde bucal e utilização de serviços odontológicos entre adolescentes da sétima série da rede pública municipal de Gravataí, Rio Grande do Sul, Brasil, no ano de 2005.

\section{Método}

Trata-se de um estudo transversal, investigando uma amostra representativa de 1.170 adolescentes, entre 12 a 18 anos, da rede pública municipal de Gravataí. O município, integrante da Região Metropolitana de Porto Alegre, possui uma população de 270.763 habitantes, sendo a quinta economia do estado e a segunda maior rede municipal de ensino do Rio Grande do Sul (Prefeitura Municipal de Gravataí; http://www.gravatai. rs.gov.br, acessado em 12/Dez/2007). No total, são 65 escolas municipais distribuídas nas áreas rural e urbana que atendiam cerca de 23 mil alunos em 2005, e todas foram incluídas na seleção da amostra.
Os participantes do estudo foram selecionados em um processo de amostragem por conglomerado em uma única etapa. No cálculo da população em estudo, foram tomados, como referência, a população alvo (2.282 alunos da sétima série), maior estimativa de prevalência dos diferentes desfechos (50\%), nível de 95\% de confiança e erro máximo tolerado de $\pm 3 \%$, estimando-se uma amostra de 728 sujeitos. Considerando um efeito de delineamento de 1,5 , acrescido de $20 \%$ para possíveis perdas, chegou-se ao número de 1.312 escolares. Como esse representava, aproximadamente, metade dos alunos matriculados, decidiu-se selecionar aleatoriamente metade mais uma das turmas nas 15 regiões administrativas de Gravataí, garantindo a proporcionalidade de escolares por turma, considerando que cada uma tinha entre 30 e 35 integrantes. Em cada turma sorteada, todos os alunos foram selecionados, totalizando 1.366 adolescentes.

Ao longo da coleta de dados, ocorreram 196 perdas, resultantes de recusa ao preenchimento dos questionários (30), não autorização à participação na pesquisa (31), transferência de escola (105), ausência no dia da aplicação dos questionários (17), evasão escolar (12) e expulsão (1). Essas perdas corresponderam a $14,3 \%$, tendo-se distribuído proporcionalmente entre meninos e meninas e nas regiões administrativas de Gravataí. Ao final, a amostra foi composta por 1.170 escolares, tendo um poder de detectar razões de prevalência iguais ou superiores a 2,0, para fatores em estudo com uma prevalência de até $10 \%$.

A coleta dos dados foi realizada nas escolas, utilizando questionários de autopreenchimento que foram aplicados por pesquisadores treinados para o esclarecimento de dúvidas. O primeiro instrumento continha informações relativas a hábitos alimentares, data de nascimento, sexo e condições sócio-econômicas familiares. O segundo foi o Global School-Based Student Health Survey (GSHS) 15, questionário para investigação da saúde do escolar desenvolvido pela OMS, do qual foram extraídos dados sobre uso, na vida, de cigarro, bebidas alcoólicas e outras drogas (maconha, cocaína, crack, ecstasy, solventes, anfetaminas e anabolizantes), experiências em casa e na escola e sentimentos depressivos. Para investigação da saúde bucal, foram incluídas, nesse instrumento, questões adaptadas de outros estudos sobre o assunto. A informação sobre cor da pele auto-referida foi obtida da Ficha de Avaliação Antropométrica, instrumento utilizado por outros projetos do estudo maior sobre a saúde desses escolares.

No presente estudo, foram investigados quatro desfechos: freqüência de escovação dentária $\geq 3$ vezes/dia (não; sim), uso diário de fio dental 
(não; sim), utilização anual de serviços odontológicos (não: quando < 1 visita/ano ou não vai; sim: quando $\geq 1$ visita/ano) e motivo preventivo de visita ao serviço (não: quando tratamento curativo; sim: quando tratamento preventivo).

Neste estudo, para a variável escovação dentária, optou-se pelo ponto de corte de, no mínimo, três vezes ao dia por tratar-se de adolescentes, faixa etária em que há maior vulnerabilidade para as doenças bucais 3 , na qual o autocuidado e os comportamentos protetores são mais negligenciados.

A freqüência de visitas ao serviço deve ser definida de acordo com cada situação, mas a recomendação de visitas anuais justifica-se pela maior probabilidade de detecção precoce de doenças bucais 17,18.

As variáveis independentes foram agrupadas em sócio-demográficas, psicossociais e relacionadas ao estilo de vida. As sócio-demográficas incluem sexo, cor da pele auto-referida (agrupada em branca e não branca), inserção sócioeconômica e tipo de serviço utilizado (privado, não privado, não utiliza). A definição da inserção sócio-econômica baseou-se na classificação da Associação Brasileira de Empresas de Pesquisa (ABEP) 19, que adota cinco categorias maiores: A, B, C, D e E. Os escolares classificados nas categorias D e E foram agrupados, em função de que apenas seis pertenciam à categoria E. Além disso, foi excluída a categoria A pela não existência de escolares na mesma.

Nas análises multivariadas para os desfechos "utilização anual de serviços odontológicos" e "motivo preventivo de visita ao serviço", os 126 escolares que referiram não fazer uso de serviços odontológicos foram excluídos. Entretanto, para o estudo dos fatores associados à freqüência de escovação dentária e uso diário de fio dental, todos os escolares participaram da análise.

Os fatores psicossociais estão compostos pelos blocos de variáveis relacionadas ao apoio social (freqüência com que o escolar foi compreendido pelos pais e número de amigos próximos), sentimentos depressivos (dificuldade para dormir, sentimento de solidão e planejamento suicida) e sentimento de discriminação, quando o escolar referiu sentir-se maltratado ou discriminado pela sua raça, cor, religião, aparência física ou outras razões. As questões relativas ao apoio social referiram-se aos últimos trinta dias, e as relacionadas aos sentimentos depressivos e discriminação, aos últimos 12 meses, conforme orientação da OMS 15.

Os fatores relacionados ao estilo de vida referem-se ao consumo de doces e uso, na vida, de tabaco, bebidas alcoólicas e outras drogas. A variável consumo de doces considera se, entre os alimentos que o adolescente refere consumir com maior freqüência, os doces estão incluídos.

Os dados foram analisados por regressão de Cox modificada para estudos transversais, utilizando-se o programa Stata 6.0 (Stata Corp., College Station, Estados Unidos). Inicialmente, investigou-se a existência de associação entre os desfechos e os fatores em estudo por meio das análises univariadas exploratórias. A seguir, realizou-se quatro análises multivariadas, uma para cada desfecho, utilizando o modelo conceitual hierarquizado concebido a priori (Figura 1).

O modelo utilizado na análise foi concebido pelos autores e apoiado na literatura sobre modelos hierárquicos 20 , apresentando quatro etapas que estabelecem a hierarquia de influência dos fatores estudados sobre os desfechos. $\mathrm{Na}$ primeira etapa, foram introduzidas as variáveis sexo, cor da pele e inserção sócio-econômica. Na segunda etapa, foram introduzidas as variáveis pertencentes ao bloco do apoio social. Nesse nível hierárquico, também foi incluída a variável correspondente ao tipo de serviço que o escolar costuma consultar. Na terceira etapa, houve a introdução da variável "sentimento de discriminação" e das variáveis relacionadas a sentimentos depressivos. Na última, ingressaram, no modelo, as relacionadas ao estilo de vida.

O ponto de corte estabelecido para seleção das variáveis que passariam à etapa seguinte foi um nível de significância $\leq 0,10$. Procedeu-se, assim, para evitar a retirada de variáveis do modelo que poderiam atuar como fatores de confusão. Uma vez selecionada a variável, mesmo que perdesse significância nas etapas posteriores, permanecia no modelo, contribuindo com razão de prevalência, intervalo de confiança e nível de significância encontrados na etapa em que foi selecionada. Foram consideradas associações estatisticamente significativas razões de prevalência com valores de $\mathrm{p}<0,05$.

Este trabalho é um dos subprojetos do estudo A Saúde do Escolar da Rede Pública Municipal de Gravataí/RS, aprovado pelo Conselho de Ética em Pesquisa da Universidade Luterana do Brasil (ULBRA; 2004-375H), seguindo as recomendações da Resolução no. 196/96 do Conselho Nacional de Saúde. Aos pais ou responsáveis pelos alunos foi solicitada a assinatura do Termo de Consentimento Livre Esclarecido. 


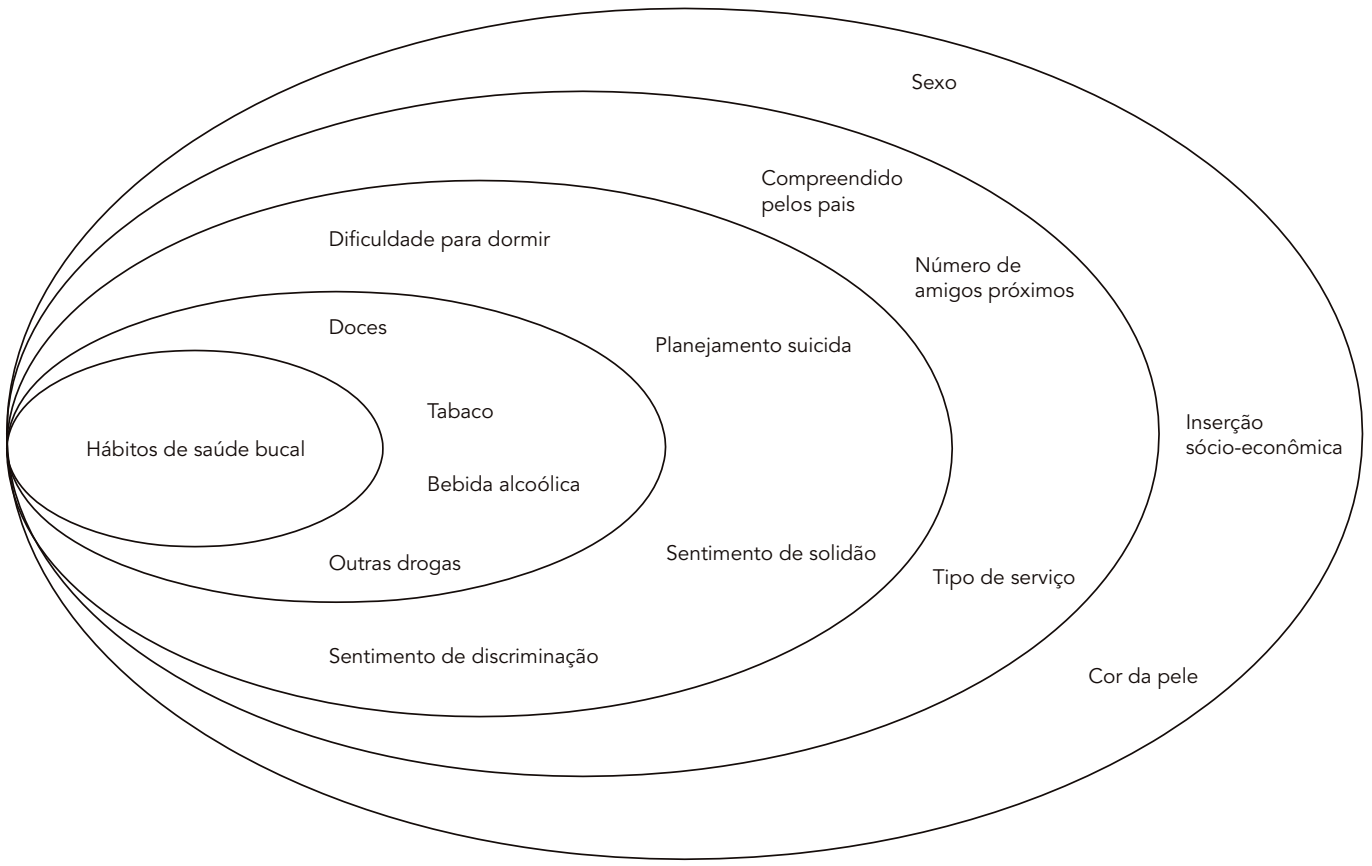

\section{Resultados}

Entre os 1.170 escolares investigados, 614 (52,5\%) pertenciam ao sexo feminino, e 615 (52,6\%) referiram cor da pele branca. Em relação à inserção sócio-econômica, $256(21,9 \%)$ estavam inseridos na categoria B; 688 (58,8\%), na categoria C; e 226 (19,3\%), na D+E (Tabela 1). A idade média dos adolescentes foi de 14 anos (desvio-padrão-DP = 1,13 ano).

A maioria dos escolares realizava escovação com uma freqüência de, pelo menos, 3 vezes/dia $(77,8 \%)$, enquanto que apenas $31,9 \%$ faziam uso diário de fio dental. Observou-se que 68,9\% dos adolescentes consultavam, no mínimo, uma vez ao ano e, para $45 \%$, o motivo da consulta foi a busca de tratamento preventivo. As Tabelas $1 \mathrm{e}$ 2 apresentam os resultados das análises univariadas e a distribuição dos escolares segundo as categorias dos fatores em estudo para os quatro desfechos investigados.

$\mathrm{Na}$ análise univariada, o desfecho freqüência de escovação dentária $\geq 3$ vezes/dia associou-se com sexo e tipo de serviço utilizado; o uso diário de fio dental mostrou associação com inserção sócio-econômica, tipo de serviço utilizado, compreensão dos pais e sentimento de solidão (Tabela 1).

$\mathrm{Na}$ análise multivariada, a freqüência de escovação dentária apresentou associação estatisticamente significativa com sexo, mostrando que as meninas tiveram uma freqüência de escovação $\geq 3$ vezes/dia $18 \%$ mais elevada do que os meninos. Também houve associação significativa entre esse desfecho e os sentimentos de discriminação e de solidão (Tabela 3). Os adolescentes que não se sentiam discriminados e os que não manifestaram sentimento de solidão informaram, respectivamente, uma prevalência $10 \%$ e 7\% maior de escovação dentária.

O uso diário de fio dental mostrou-se associado, significativamente, com a inserção sócio-econômica, apresentando prevalências 34\% e 51\% maiores nas categorias $\mathrm{C}$ e $\mathrm{B}$, respectivamente, quando comparadas à categoria $\mathrm{D}+\mathrm{E}$. Os jovens que freqüentavam os serviços privados referiram $83 \%$ mais uso de fio dental do que aqueles que não faziam uso de serviços odontológicos (Tabela 3).

A freqüência com que o adolescente sentiuse compreendido pelos pais também se associou com o uso diário de fio dental, sendo 39\% maior 
Resultado da análise univariada com os desfechos freqüência de escovação dentária e uso diário de fio dental. Gravataí, Rio Grande do Sul, Brasil, 2005.

\begin{tabular}{|c|c|c|c|c|c|c|c|c|c|}
\hline \multirow[t]{2}{*}{ Variáveis } & \multirow[t]{2}{*}{$\mathbf{N}$} & \multicolumn{4}{|c|}{ Freqüência de escovação dentária $\geq 3$ vezes/dia } & \multicolumn{4}{|c|}{ Uso diário de fio dental } \\
\hline & & $\mathrm{n}$ & $\%$ & RP (IC95\%) & $\begin{array}{l}\text { Valor } \\
\text { de } p\end{array}$ & $\mathbf{n}$ & $\%$ & RP (IC95\%) & $\begin{array}{l}\text { Valor } \\
\text { de } p\end{array}$ \\
\hline \multicolumn{10}{|l|}{ Sexo } \\
\hline Masculino & 556 & 395 & 71,0 & 1,00 & - & 167 & 30,0 & 1,00 & - \\
\hline Feminino & 614 & 515 & 83,9 & $1,18(1,11-1,25)$ & 0,000 & 206 & 33,5 & $1,11(0,94-1,32)$ & 0,199 \\
\hline \multicolumn{10}{|l|}{ Cor da pele } \\
\hline Não branca & 555 & 438 & 78,9 & 1,00 & - & 171 & 30,8 & 1,00 & - \\
\hline Branca & 615 & 472 & 76,7 & $0,97(0,91-1,03)$ & 0,372 & 202 & 32,8 & $1,06(0,90-1,26)$ & 0,457 \\
\hline \multicolumn{10}{|l|}{ Inserção sócio-econômica } \\
\hline$D+E$ & 226 & 170 & 75,2 & 1,00 & - & 55 & 24,3 & 1,00 & - \\
\hline C & 688 & 541 & 78,6 & $1,04(0,96-1,13)$ & 0,303 & 224 & 32,6 & $1,33(1,03-1,72)$ & 0,025 \\
\hline B & 256 & 199 & 77,7 & $1,03(0,93-1,14)$ & 0,518 & 94 & 36,7 & $1,50(1,13-1,99)$ & 0,004 \\
\hline \multicolumn{10}{|l|}{ Tipo de serviço } \\
\hline Não utiliza & 126 & 89 & 70,6 & 1,00 & - & 27 & 21,4 & 1,00 & - \\
\hline Não privado & 783 & 609 & 77,8 & $1,10(0,97-1,23)$ & 0,112 & 235 & 30,0 & 1,40 (0,98-1,98 & 0,060 \\
\hline Privado & 261 & 212 & 81,2 & $1,14(1,01-1,30)$ & 0,031 & 111 & 42,5 & $1,98(1,38-2,85)$ & 0,000 \\
\hline \multicolumn{10}{|l|}{ Compreendido pelos pais } \\
\hline Nunca & 260 & 195 & 75,0 & 1,00 & - & 65 & 25,0 & 1,00 & - \\
\hline Raramente/Às vezes & 309 & 230 & 74,4 & $0,99(0,90-1,09)$ & 0,877 & 90 & 29,1 & $1,16(0,88-1,53)$ & 0,273 \\
\hline Sempre/Quase sempre & 601 & 485 & 80,7 & $1,07(0,99-1,16)$ & 0,074 & 218 & 36,3 & $1,45(1,14-1,83)$ & 0,002 \\
\hline \multicolumn{10}{|l|}{ Amigos próximos } \\
\hline Nenhum, 1 ou 2 & 183 & 136 & 74,3 & 1,00 & - & 60 & 32,8 & 1,00 & - \\
\hline 3 ou mais & 986 & 773 & 78,4 & $1,05(0,96-1,15)$ & 0,251 & 313 & 31,7 & $0,96(0,77-1,21)$ & 0,780 \\
\hline \multicolumn{10}{|l|}{ Sentimento discriminação } \\
\hline Sim & 246 & 181 & 73,6 & 1,00 & - & 70 & 24,5 & 1,00 & - \\
\hline Não & 924 & 729 & 78,9 & $1,07(0,98-1,16)$ & 0,095 & 303 & 32,8 & $1,15(0,92-1,43)$ & 0,204 \\
\hline \multicolumn{10}{|l|}{ Dificuldade para dormir } \\
\hline Sempre/Quase sempre & 34 & 26 & 76,5 & 1,00 & - & 10 & 29,4 & 1,00 & - \\
\hline Raramente/Às vezes & 425 & 336 & 79,1 & $1,03(0,85-1,25)$ & 0,735 & 118 & 27,8 & $0,94(0,54-1,62)$ & 0,835 \\
\hline Nunca & 710 & 547 & 77,0 & $1,00(0,83-1,21)$ & 0,939 & 245 & 34,5 & $1,17(0,69-1,99)$ & 0,555 \\
\hline \multicolumn{10}{|l|}{ Sentimento de solidão } \\
\hline Sim & 598 & 457 & 76,4 & 1,00 & - & 161 & 27,0 & 1,00 & - \\
\hline Não & 571 & 452 & 79,1 & $1,03(0,97-1,10)$ & 0,260 & 212 & 37,1 & $1,38(1,16-1,63)$ & 0,000 \\
\hline \multicolumn{10}{|l|}{ Planejamento suicida } \\
\hline Sim & 74 & 54 & 73,0 & 1,00 & - & 22 & 29,7 & 1,00 & - \\
\hline Não & 1095 & 855 & 78,1 & $1,07(0,92-1,23)$ & 0,351 & 351 & 32,0 & $1,07(0,75-1,54)$ & 0,683 \\
\hline \multicolumn{10}{|l|}{ Consumo de doces } \\
\hline Sim & 772 & 595 & 77,1 & 1,00 & - & 248 & 32,1 & 1,00 & - \\
\hline Não & 385 & 304 & 79,0 & 1,24 (0,96-1,09) & 0,461 & 122 & 31,7 & $0,98(0,82-1,17)$ & 0,881 \\
\hline \multicolumn{10}{|l|}{ Consumo de tabaco } \\
\hline Sim & 195 & 151 & 77,4 & 1,00 & - & 51 & 26,1 & 1,00 & - \\
\hline Não & 975 & 759 & 77,8 & 1,00 (0,92-1,09) & 0,901 & 322 & 33,0 & $1,26(0,98-1,62)$ & 0,070 \\
\hline \multicolumn{10}{|c|}{ Consumo de bebida alcoólica } \\
\hline Sim & 710 & 548 & 77,2 & 1,00 & - & 217 & 30,6 & 1,00 & - \\
\hline Não & 460 & 362 & 78,7 & $1,01(0,95-1,08)$ & 0,541 & 156 & 33,9 & $1,10(0,93-1,31)$ & 0,228 \\
\hline \multicolumn{10}{|l|}{ Consumo de outras drogas } \\
\hline Sim & 27 & 22 & 81,5 & 1,00 & - & 9 & 33,3 & 1,00 & - \\
\hline Não & 1.143 & 888 & 77,7 & $0,95(0,79-1,14)$ & 0,609 & 364 & 31,8 & $0,95(0,55-1,64)$ & 0,869 \\
\hline
\end{tabular}

RP: razão de prevalência. 
Tabela 2

Resultado da análise univariada com os desfechos utilização anual de serviços odontológicos e motivo preventivo de visita ao serviço. Gravataí, Rio Grande do Sul, Brasil, 2005.

\begin{tabular}{|c|c|c|c|c|c|c|c|c|}
\hline \multirow[t]{2}{*}{ Variáveis } & \multicolumn{4}{|c|}{ Utilização anual de serviços odontológicos } & \multicolumn{4}{|c|}{ Motivo preventivo de visita ao serviço } \\
\hline & $\mathbf{n}$ & $\%$ & RP (IC95\%) & Valor de $p$ & $\mathrm{n}$ & $\%$ & RP (IC95\%) & Valor de $p$ \\
\hline \multicolumn{9}{|l|}{ Sexo } \\
\hline Masculino & 376 & 67,6 & 1,00 & - & 225 & 45,0 & 1,00 & - \\
\hline Feminino & 430 & 70,0 & $1,03(0,95-1,11)$ & 0,376 & 255 & 45,0 & $1,00(0,87-1,14)$ & 0,986 \\
\hline \multicolumn{9}{|l|}{ Cor da pele } \\
\hline Não branca & 372 & 60,0 & 1,00 & - & 220 & 43,6 & 1,00 & - \\
\hline Branca & 434 & 70,6 & $1,05(0,97-1,13)$ & 0,193 & 260 & 46,3 & $1,06(0,93-1,21)$ & 0,363 \\
\hline \multicolumn{9}{|l|}{ Inserção sócio-econômica } \\
\hline$D+E$ & 122 & 54,0 & 1,00 & & 60 & 30,9 & 1,00 & - \\
\hline C & 483 & 70,2 & $1,30(1,14-1,48)$ & 0,000 & 287 & 45,7 & $1,47(1,17-1,85)$ & 0,001 \\
\hline B & 201 & 78,5 & $1,45(1,26-1,66)$ & 0,000 & 133 & 54,5 & $1,76(1,38-2,23)$ & 0,000 \\
\hline \multicolumn{9}{|l|}{ Tipo de serviço utilizado } \\
\hline Não privado & 589 & 75,2 & 1,00 & & 339 & 43,5 & 1,00 & - \\
\hline Privado & 211 & 80,8 & $1,07(1,00-1,15)$ & 0,048 & 130 & 49,1 & $1,14(0,98-1,32)$ & 0,069 \\
\hline \multicolumn{9}{|l|}{ Compreendido pelos pais } \\
\hline Nunca & 166 & 66,8 & 1,00 & - & 71 & 33,6 & 1,00 & - \\
\hline Raramente/Às vezes & 208 & 67,3 & $1,05(0,93-1,18)$ & 0,388 & 124 & 44,9 & $1,33(1,07-1,66)$ & 0,011 \\
\hline Sempre/Quase sempre & 432 & 71,9 & $1,12(1,01-1,24)$ & 0,026 & 277 & 49,9 & $1,48(1,21-1,81)$ & 0,000 \\
\hline \multicolumn{9}{|l|}{ Amigos próximos } \\
\hline Nenhum, 1 ou 2 & 125 & 68,3 & 1,00 & - & 72 & 42,6 & 1,00 & - \\
\hline 3 ou mais & 681 & 69,1 & $1,01(0,90-1,12)$ & 0,840 & 408 & 45,5 & $1,06(0,88-1,29)$ & 0,490 \\
\hline \multicolumn{9}{|c|}{ Sentimento de discriminação } \\
\hline Sim & 158 & 64,2 & 1,00 & - & 105 & 46,2 & 1,00 & - \\
\hline Não & 648 & 70,1 & $1,09(0,98-1,20)$ & 0,092 & 375 & 44,7 & $0,96(0,82-1,13)$ & 0,673 \\
\hline \multicolumn{9}{|l|}{ Dificuldade para dormir } \\
\hline Sempre/Quase sempre & 18 & 52,9 & 1,00 & - & 15 & 48,4 & 1,00 & - \\
\hline Raramente/Às vezes & 292 & 68,7 & $1,29(0,93-1,79)$ & 0,114 & 179 & 46,4 & $0,95(0,65-1,40)$ & 0,826 \\
\hline Nunca & 496 & 69,9 & $1,31(0,95-1,81)$ & 0,090 & 286 & 44,1 & $0,91(0,62-1,32)$ & 0,630 \\
\hline \multicolumn{9}{|l|}{ Sentimento de solidão } \\
\hline Sim & 397 & 66,4 & 1,00 & - & 241 & 44,3 & 1,00 & - \\
\hline Não & 409 & 71,6 & $1,08(0,10-1,16)$ & 0,053 & 239 & 45,9 & $1,03(0,91-1,18)$ & 0,606 \\
\hline \multicolumn{9}{|l|}{ Planejamento suicida } \\
\hline Sim & 49 & 66,2 & 1,00 & - & 26 & 39,3 & 1,00 & - \\
\hline Não & 757 & 69,1 & $1,04(0,88-1,23)$ & 0,614 & 454 & 45,4 & $1,15(0,84-1,56)$ & 0,362 \\
\hline \multicolumn{9}{|l|}{ Consumo de doces } \\
\hline Sim & 532 & 68,9 & 1,00 & - & 297 & 41,9 & 1,00 & - \\
\hline Não & 264 & 65,6 & $0,99(0,91-1,08)$ & 0,906 & 176 & 50,7 & $1,21(1,05-1,38)$ & 0,006 \\
\hline \multicolumn{9}{|l|}{ Consumo de tabaco } \\
\hline Sim & 119 & 61,0 & 1,00 & - & 64 & 36,1 & 1,00 & - \\
\hline Não & 687 & 70,5 & $1,15(1,02-1,30)$ & 0,018 & 416 & 46,8 & $1,29(1,05-1,59)$ & 0,015 \\
\hline \multicolumn{9}{|c|}{ Consumo de bebida alcoólica } \\
\hline Sim & 487 & 68,6 & 1,00 & - & 288 & 44,6 & 1,00 & - \\
\hline Não & 319 & 69,3 & $1,01(0,93-1,09)$ & 0,784 & 192 & 45,7 & $1.02(0,89-1,17)$ & 0,716 \\
\hline \multicolumn{9}{|l|}{ Consumo de outras drogas } \\
\hline Sim & 17 & 63,0 & 1,00 & - & 8 & 36,4 & 1,00 & - \\
\hline Não & 789 & 69,0 & $1,09(0,81-1,46)$ & 0,537 & 472 & 45,2 & $1,24(0,71-2,17)$ & 0,444 \\
\hline
\end{tabular}

RP: razão de prevalência. 
Resultado da análise multivariada com os desfechos freqüência de escovação dentária e uso diário de fio dental. Gravataí, Rio Grande do Sul, Brasil, 2005.

\begin{tabular}{|c|c|c|c|c|c|c|}
\hline \multirow[t]{2}{*}{ Variáveis } & \multicolumn{3}{|c|}{ Freqüência de escovação dentária $\geq 3$ vezes/dia } & \multicolumn{3}{|c|}{ Uso diário de fio dental } \\
\hline & RP & $\mathrm{IC} 95 \%$ & Valor de $p$ & RP & IC95\% & Valor de $p$ \\
\hline \multicolumn{7}{|l|}{ Sexo } \\
\hline Masculino & 1,00 & - & - & 1,00 & - & - \\
\hline Feminino & 1,18 & $1,11-1,26$ & 0,000 & 1,13 & $0,95-1,34$ & 0,149 \\
\hline \multicolumn{7}{|l|}{ Cor da pele } \\
\hline Não branca & 1,00 & - & - & 1,00 & - & - \\
\hline Branca & 0,96 & $0,90-1,02$ & 0,242 & 1,04 & $0,88-1,23$ & 0,624 \\
\hline \multicolumn{7}{|l|}{ Inserção sócio-econômica } \\
\hline$D+E$ & 1,00 & - & - & 1,00 & - & - \\
\hline C & 1,05 & $0,97-1,15$ & 0,191 & 1,34 & $1,04-1,73$ & 0,023 \\
\hline B & 1,05 & $0,95-1,16$ & 0,300 & 1,51 & $1,14-2,01$ & 0,004 \\
\hline \multicolumn{7}{|l|}{ Tipo de serviço utilizado } \\
\hline Não utiliza & 1,00 & $0,96-1,22$ & - & 1,00 & - & - \\
\hline Não privado & 1,08 & $0,99-1,28$ & 0,180 & 1,35 & $0,95-1,93$ & 0,091 \\
\hline Privado & 1,13 & - & 0,064 & 1,83 & $1,27-2,65$ & 0,001 \\
\hline \multicolumn{7}{|l|}{ Compreendido pelos pais } \\
\hline Nunca & 1,00 & - & - & 1,00 & - & - \\
\hline Raramente/Às vezes & 0,98 & $0,89-1,07$ & 0,638 & 1,14 & $0,86-1,49$ & 0,358 \\
\hline Sempre/Quase sempre & 1,05 & $0,97-1,14$ & 0,196 & 1,39 & $1,10-1,76$ & 0,006 \\
\hline \multicolumn{7}{|l|}{ Amigos próximos } \\
\hline Nenhum, 1 ou 2 & 1,00 & - & - & 1,00 & - & - \\
\hline 3 ou mais & 1,06 & $0,97-1,16$ & 0,198 & 0,96 & $0,77-1,20$ & 0,709 \\
\hline \multicolumn{7}{|l|}{ Sentimento de discriminação } \\
\hline Sim & 1,00 & - & - & 1,00 & - & 0,946 \\
\hline Não & 1,10 & $1,01-1,19$ & 0,047 & 1,00 & $0,80-1,27$ & - \\
\hline \multicolumn{7}{|l|}{ Dificuldade para dormir } \\
\hline Sempre/Quase sempre & 1,00 & - & - & 1,00 & - & - \\
\hline Raramente/Às vezes & 1,01 & $0,84-1,24$ & 0,855 & 0,89 & $0,52-1,53$ & 0,671 \\
\hline Nunca & 1,00 & $0,97-1,19$ & 0,797 & 0,99 & $0,58-1,69$ & 0,964 \\
\hline \multicolumn{7}{|l|}{ Sentimento de solidão } \\
\hline Sim & 1,00 & - & - & 1,00 & - & - \\
\hline Não & 1,07 & $1,01-1,15$ & 0,047 & 1,30 & $1,08-1,58$ & 0,006 \\
\hline \multicolumn{7}{|l|}{ Planejamento suicida } \\
\hline $\operatorname{Sim}$ & 1,00 & - & - & 1,00 & - & - \\
\hline Não & 1,06 & $0,92-1,23$ & 0,383 & 0,93 & $0,64-1,34$ & 0,7111 \\
\hline \multicolumn{7}{|l|}{ Consumo de doces } \\
\hline Sim & 1,00 & - & - & 1,00 & - & - \\
\hline Não & 1,04 & $0,98-1,11$ & 0,213 & 1,01 & $0,84-1,20$ & 0,071 \\
\hline \multicolumn{7}{|l|}{ Consumo de tabaco } \\
\hline Sim & 1,00 & - & - & 1,00 & - & - \\
\hline Não & 0,99 & $0,90-1,09$ & 0,878 & 1,18 & $0,90-1,54$ & 0,232 \\
\hline \multicolumn{7}{|l|}{ Consumo de bebida alcoólica } \\
\hline Sim & 1,00 & - & - & 1,00 & - & - \\
\hline Não & 1,00 & $0,94-1,07$ & 0,850 & 1,06 & $0,89-1,23$ & 0,654 \\
\hline \multicolumn{7}{|l|}{ Consumo de outras drogas } \\
\hline Sim & 1,00 & - & - & 1,00 & - & - \\
\hline Não & 0,93 & $0,76-1,13$ & 0,457 & 0,76 & $0,44-1,30$ & 0,315 \\
\hline
\end{tabular}

RP: razão de prevalência. 
entre os que sempre ou quase sempre se sentiam compreendidos. Em relação ao sentimento de solidão, esse desfecho foi $30 \%$ mais freqüente entre os que responderam não se sentirem solitários (Tabela 3).

Conforme a Tabela 2, a utilização anual de serviços odontológicos e motivo preventivo de visita ao serviço estiveram associados à inserção sócio-econômica, compreensão dos pais e consumo de tabaco. No entanto, além desses, o primeiro desfecho associou-se, também, ao tipo de serviço utilizado, e o segundo, ao consumo de doces.

Após o ajuste, a utilização anual de serviços odontológicos somente manteve associação com inserção sócio-econômica, sendo 30\% maior entre os jovens da categoria C e $45 \%$ maior entre os da B, quando comparados aos da categoria D+E (Tabela 4).

O motivo da visita ao serviço também apresentou associação estatisticamente significativa com inserção sócio-econômica, sendo a procura por tratamento preventivo $47 \%$ e $75 \%$ maior nas categorias $\mathrm{C}$ e B, respectivamente, do que na categoria $\mathrm{D}+\mathrm{E}$. Ocorrendo o mesmo em relação à variável que investigou se o adolescente sentia-se compreendido pelos pais. Observou-se uma prevalência $32 \%$ maior de busca de consultas preventivas entre os que se sentiam compreendidos raramente ou às vezes e $41 \%$ maior entre os que sempre ou quase sempre se sentiam assim, comparados aos que informaram nunca se sentirem compreendidos pelos pais. Entre os jovens que não costumavam consumir doces, encontrou-se uma prevalência de visitas ao serviço para tratamento preventivo de $21 \%$ (Tabela 4 ).

\section{Discussão}

Este é um estudo de base escolar e, como tal, requer alguns cuidados em relação à interpretação de seus achados. Os adolescentes de escolas privadas e os que estão fora da escola não foram investigados. Assim, os resultados não podem ser tomados como representativos de todos os adolescentes de Gravataí. Além disso, apesar de terem sido utilizados questionários auto-aplicáveis e assegurada a confidencialidade dos dados, deve-se considerar a possibilidade de ter ocorrido algum viés de informação. Contudo, acredita-se que os resultados possam ser extrapolados para outros jovens de escolas públicas da Região Metropolitana de Porto Alegre, em função das características semelhantes existentes entre as cidades.

Entre os jovens estudados, a freqüência de escovação dentária mostrou-se alta, similar a outros estudos 21,22, sendo significativamente maior entre as adolescentes, como já era esperado. Diferenças relacionadas à adoção de hábitos e comportamentos de saúde entre os gêneros vêm sendo observadas, identificando que as mulheres adotam mais comportamentos positivos relacionados à saúde do que os homens 6,22. Além disso, elas têm maior preocupação com sua aparência física, muito influenciada pelos padrões sociais e culturais vigentes, contribuindo para que assumam maior cuidado em relação ao seu corpo, repercutindo também nos hábitos e comportamentos de saúde bucal 6 .

Os adolescentes que não se sentiram discriminados também relataram maior freqüência de escovação dentária do que aqueles que se sentiram assim, revelando maior cuidado consigo mesmos e com sua saúde, talvez em conseqüência da aceitação e identificação com o grupo de convívio. Vítimas de discriminação, em geral, apresentam baixa auto-estima 23 , o que pode influenciar negativamente os seus hábitos de saúde. Aqueles que não se sentiam solitários também escovavam os dentes com maior freqüência, provavelmente por sentirem-se mais motivados para realizarem higiene bucal. Avlund et al. 7 observaram que pessoas que vivem sozinhas ou que não estão satisfeitas com a freqüência de seus contatos sociais adotam menos comportamentos preventivos de saúde e têm maior ocorrência de cáries radiculares.

Quanto ao uso diário de fio dental, esse foi maior entre os adolescentes de inserção sócioeconômica mais alta e entre os que utilizavam os serviços privados. A condição sócio-econômica é um fator diretamente relacionado aos cuidados com a saúde bucal. Estudos demonstram que indivíduos pertencentes a categorias sócio-econômicas mais elevadas escovam mais seus dentes, utilizam fio dental e serviços de saúde com maior freqüência do que os de inserção sócio-econômica mais baixa 21,24. Além disso, os usuários de serviços privados têm maior probabilidade de receberem instruções de higiene bucal no consultório 25 e de terem poder aquisitivo para adquirir fio dental, o que facilitaria a adoção desse hábito. Como foram investigados escolares da rede pública, é provável que, para a maioria deles, o custo dos serviços privados e a aquisição de fio dental sejam onerosos, o que explicaria o resultado encontrado.

No caso específico da população investigada, deve-se considerar o fato de que as escolas orientam os alunos sobre higiene bucal, incluindo a distribuição de escova dental. O fio dental, no entanto, não é oferecido. É possível que essa seja a explicação para a alta freqüência de escovação, não ocorrendo o mesmo em relação ao uso de 
Resultado da análise multivariada com os desfechos utilização anual de serviços odontológicos e motivo preventivo de visita ao serviço. Gravataí, Rio Grande do Sul, Brasil, 2005.

\begin{tabular}{|c|c|c|c|c|c|c|}
\hline \multirow[t]{2}{*}{ Variáveis } & \multicolumn{3}{|c|}{ Utilização anual de serviços odontológicos } & \multicolumn{3}{|c|}{ Motivo preventivo de visita ao serviço } \\
\hline & RP & IC95\% & Valor de $\mathrm{p}$ & RP & IC95\% & Valor de $\mathrm{p}$ \\
\hline \multicolumn{7}{|l|}{ Sexo } \\
\hline Masculino & 1,00 & - & - & 1,00 & - & - \\
\hline Feminino & 1,04 & $0,97-1,13$ & 0,223 & 1,01 & $0,89-1,16$ & 0,796 \\
\hline \multicolumn{7}{|l|}{ Cor da pele } \\
\hline Não branca & 1,00 & - & - & 1,00 & - & - \\
\hline Branca & 1,03 & $0,95-1,11$ & 0,387 & 1,03 & $0,91-1,18$ & 0,566 \\
\hline \multicolumn{7}{|l|}{ Inserção sócio-econômica } \\
\hline $\mathrm{D}+\mathrm{E}$ & 1,00 & - & - & 1,00 & - & - \\
\hline C & 1,30 & $1,14-1,48$ & 0,000 & 1,47 & $1,17-1,85$ & 0,001 \\
\hline B & 1,45 & $1,26-1,66$ & 0,000 & 1,75 & $1,38-2,23$ & 0,000 \\
\hline \multicolumn{7}{|l|}{ Tipo de serviço utilizado } \\
\hline Não privado & 1,00 & - & - & 1,00 & - & - \\
\hline Privado & 1,03 & $0,97-1,12$ & 0,299 & 1,04 & $0,80-1,21$ & 0,555 \\
\hline \multicolumn{7}{|l|}{ Compreendido pelos pais } \\
\hline Nunca & 1,00 & - & - & 1,00 & - & - \\
\hline Raramente/Às vezes & 1,03 & $0,93-1,14$ & 0,547 & 1,32 & $1,05-1,64$ & 0,015 \\
\hline Sempre/Quase sempre & 1,07 & $0,98-1,17$ & 0,143 & 1,41 & $1,16-1,72$ & 0,001 \\
\hline \multicolumn{7}{|l|}{ Amigos próximos } \\
\hline Nenhum, 1 ou 2 & 1,00 & - & - & 1,00 & - & - \\
\hline 3 ou mais & 1,02 & $0,93-1,12$ & 0,653 & 1,01 & $0,84-1,22$ & 0,862 \\
\hline \multicolumn{7}{|l|}{ Sentimento de discriminação } \\
\hline Sim & 1,00 & - & - & 1,00 & - & - \\
\hline Não & 1,06 & $0,96-1,19$ & 0,249 & 0,96 & $0,81-1,14$ & 0,645 \\
\hline \multicolumn{7}{|l|}{ Dificuldade para dormir } \\
\hline Sempre/Quase sempre & 1,00 & - & - & 1,00 & - & - \\
\hline Raramente/Às vezes & 1,26 & $0,91-1,74$ & 0,170 & 0,83 & $0,57-1,23$ & 0,359 \\
\hline Nunca & 1,23 & $0,89-1,71$ & 0,204 & 0,77 & $0,53-1,14$ & 0,198 \\
\hline \multicolumn{7}{|l|}{ Sentimento de solidão } \\
\hline Sim & 1,00 & - & - & 1,00 & - & - \\
\hline Não & 1,06 & $0,97-1,15$ & 0,172 & 1,06 & $0,91-1,38$ & 0,462 \\
\hline \multicolumn{7}{|l|}{ Planejamento suicida } \\
\hline Sim & 1,00 & - & - & 1,00 & - & - \\
\hline Não & 0,99 & $0,83-1,17$ & 0,903 & 1,39 & $0,83-1,57$ & 0,426 \\
\hline \multicolumn{7}{|l|}{ Consumo de doces } \\
\hline Sim & 1,00 & - & - & 1,00 & - & - \\
\hline Não & 1,00 & $0,92-1,08$ & 0,969 & 1,21 & $1,06-1,38$ & 0,005 \\
\hline \multicolumn{7}{|l|}{ Consumo de tabaco } \\
\hline Sim & 1,00 & - & - & 1,00 & 1,00 & - \\
\hline Não & 1,13 & $0,99-1,28$ & 0,063 & 1,21 & $0,97-1,50$ & 0,087 \\
\hline \multicolumn{7}{|c|}{ Consumo de bebida alcoólica } \\
\hline Sim & 1,00 & - & - & 1,00 & 1,00 & - \\
\hline Não & 0,99 & $0,91-1,07$ & 0,827 & 0,98 & $0,86-1,13$ & 0,837 \\
\hline \multicolumn{7}{|l|}{ Consumo de outras drogas } \\
\hline $\operatorname{Sim}$ & 1,00 & - & - & 1,00 & - & - \\
\hline Não & 0,99 & $0,73-1,36$ & 0,976 & 1,00 & $0,57-1,76$ & 0,990 \\
\hline
\end{tabular}

RP: razão de prevalência. 
fio dental, que foi mais baixo. Outra explicação poderia ser o fato de a escovação dentária ser um hábito mais consolidado que o uso de fio dental. A prática de distribuição de escovas dentais pode ser a responsável, também, pela não associação da escovação dentária com as condições sócioeconômicas familiares, pois reduz a influência dessas, tanto na aquisição do produto quanto no estabelecimento do hábito.

Esse fato, no entanto, ressalta a importância da intervenção da escola e a influência positiva que ela pode ter na definição de hábitos de saúde bucal na infância e adolescência 26 . O fornecimento de fio dental também poderia ser uma prática adotada como rotina, uma vez que a universalização do acesso ao mesmo é uma estratégia que vem sendo incentivada pelo Ministério da Saúde, dentro de uma abordagem coletiva de promoção da saúde e prevenção da cárie dentária 17 .

O estudo de hábitos e comportamentos de saúde não deve ficar restrito aos fatores de risco para a ocorrência de doenças, devendo abordar também aspectos familiares. Nesse sentido, verificou-se que os adolescentes que se sentiram compreendidos pelos pais apresentaram maior freqüência de uso diário de fio dental. É conhecida a importância das relações familiares e sociais na saúde bucal e sua influência nos hábitos e comportamentos do indivíduo 7,8,9.

Além dos fatores familiares, deve-se levar em conta os fatores psicossociais que contribuem para a adoção de atitudes em relação à saúde, como a identificação do indivíduo com outras pessoas, que podem ser os pais, colegas ou grupo social 1. Muitas vezes, hábitos de saúde inadequados podem indicar que o adolescente sente-se incompreendido por seus pais e assume uma postura de desinteresse pela sua aparência e autocuidado, como forma de protesto. Estudos concluíram que apoio social não satisfatório está associado com comportamentos negativos de saúde e problemas de saúde mais freqüentes 7,8. As ações de promoção da saúde com adolescentes necessitam ser fundamentadas no conhecimento das suas características emocionais e comportamentais para que realmente possam ser eficazes.

$\mathrm{O}$ fato de os adolescentes que não se sentiam solitários fazerem mais uso diário de fio dental, neste estudo, está de acordo com resultados encontrados por outros autores que observaram que os sentimentos depressivos podem interferir no autocuidado, com reflexos na saúde geral e bucal do indivíduo 27,28. O estresse psicossocial e outros fatores psicológicos que interferem no bem-estar podem ter efeitos diretos ou indiretos na etiologia da periodontite 27 . O estresse pode influenciar o comportamento de uma pessoa em relação à nutrição e higiene oral; a depressão pode reduzir a energia e a autodisciplina do indivíduo resultando em deterioração da higiene e da condição periodontal 27 . Em estudo realizado na Finlândia, os autores demonstraram que há um risco aumentado para danos à saúde bucal entre sujeitos com sintomas depressivos ou de ansiedade 28 .

O padrão de utilização dos serviços é considerado elemento importante no estudo de hábitos de saúde, pois está relacionado à necessidade de tratamento e ao autocuidado ${ }^{29}$. No presente estudo, a freqüência de utilização de serviços foi mais alta do que em outros, como o de Barros \& Bertoldi 30 , que encontraram 44,4\% em nível nacional, e o de Pinheiro et al. 18 , que identificaram 33\% no Brasil e 41,5\% no Rio Grande do Sul. É possível que isso ocorra em função de o Município de Gravataí desenvolver ações de promoção de saúde voltadas para a comunidade escolar, incentivando a busca de serviços de atenção básica para esse grupo (Prefeitura Municipal de Gravataí. Política de Atenção Integral à Saúde Bucal (PAISB); http://www.gravatai.rs.gov. br/saude/paisb.php, acessado em 12/Dez/2007). No entanto, a freqüência foi consideravelmente menor entre os escolares de inserção sócio-econômica mais baixa, como já havia sido observado em estudos anteriores 21,24.

A condição sócio-econômica é um dos determinantes sociais mais importantes na utilização de serviços odontológicos. Em estudo nacional sobre utilização de serviços, os autores observaram que o número de desassistidos era 16 vezes maior entre os mais pobres, e esses eram também os que tinham maior dificuldade de conseguir atendimento quando o buscavam ${ }^{30}$. Um dos fatores que contribui para essa situação é o fato de o Sistema Único de Saúde (SUS) ainda não ter capacidade de atender a demanda, não conseguindo suprir as necessidades de saúde bucal da população ${ }^{30}$, e também pela percepção do usuário sobre a qualidade dos serviços não privados 5, o que o levaria a não utilizá-los. Além desses, a autopercepção da saúde bucal também pode contribuir para a decisão de busca de serviços, sendo que muitos não os utilizam por não acharem necessário, mesmo quando, do ponto de vista clínico, deveriam fazê-lo 4 .

Grandes desigualdades no acesso a serviços entre os diferentes estratos sócio-econômicos foram observadas 31 . E, apesar de os autores não terem constatado que o aumento da oferta de serviços diminui as iniqüidades, puderam verificar que, com maior acesso a serviços, os grupos menos favorecidos podem ter menos perdas dentárias, pela maior possibilidade de receberem tratamento restaurador. 
Esse aspecto merece atenção especial, porque as perdas dentárias podem representar perda de oportunidades para o indivíduo, pois levam à redução da auto-estima, gerando sentimentos de rejeição e isolamento, interferindo nas relações pessoais e profissionais 32 , dificultando ainda mais a inserção dos jovens das categorias sócioeconômicas mais baixas no mercado de trabalho. Os adolescentes que freqüentam escolas públicas apresentam maior ocorrência de cárie e menor percentual de dentes restaurados 33 , estando mais sujeitos a perdas dentárias. Portanto, além de ações de promoção da saúde enfocando fatores de risco e medidas de autocuidado, políticas públicas comprometidas com a melhoria das condições sócio-econômicas são indispensáveis para propiciar mudanças na situação de saúde bucal da população adolescente.

Em relação ao motivo da visita ao serviço, menos da metade dos jovens procuraram o serviço por motivos preventivos, semelhante ao encontrado entre os jovens do Município de Canoas, Rio Grande do Sul, Brasil, em que cerca de 40\% utilizavam o serviço preventivamente 22 . Esses resultados denotam que as necessidades de tratamento curativo ainda são altas entre as populações estudadas.

Os jovens de inserção sócio-econômica mais alta procuraram mais os serviços para consultas preventivas. Isso pode ser explicado pelo fato de que aqueles que possuem melhores condições têm melhores hábitos de higiene bucal e fazem uso de serviços com maior freqüência ${ }^{20}$. Presumivelmente, também têm as suas necessidades de tratamento curativo supridas, retornando ao serviço somente para as consultas de rotina.

Os que se sentiram compreendidos pelos pais também buscaram mais os serviços para tratamento preventivo. Esse resultado parece estar diretamente relacionado ao fato de que esses adolescentes também faziam mais uso de fio dental, ou seja, adotavam mais medidas de autocuidado, resultando em melhor condição de saúde bucal. É possível, também, que esses pais se mostrem mais atentos à importância dos cuidados com a higiene de seus filhos. Assim, novamente, evidencia-se a importância do contexto familiar como determinante de saúde bucal 1,8 .

A ingestão de alimentos açucarados é considerada um fator de risco para a ocorrência de cárie dentária, que está fortemente associada à dieta 34 . Indivíduos que mantêm uma dieta com baixa quantidade de doces têm menor chance de desenvolverem a lesão. No presente estudo, os jovens que não consumiam doces procuraram mais os serviços para consultas preventivas, similar ao que ocorreu no estudo de Peres et al. 34 , no qual as crianças que consumiam menos do- ces apresentaram menor severidade de cárie e buscaram mais os serviços para tratamento preventivo. O consumo de doces foi analisado como uma variável associada ao estilo de vida, podendo demonstrar autocuidado e outros hábitos alimentares saudáveis, em um contexto de comportamentos protetores da saúde.

A cavidade bucal pode refletir as condições de vida e saúde das pessoas. Neste estudo, observou-se que a condição sócio-econômica familiar associou-se aos hábitos de saúde bucal dos adolescentes aqui investigados, com exceção à escovação dentária. Acredita-se que isso seja decorrente da distribuição de escovas feita pelas escolas, evidenciando a importância dessa estratégia.

Os fatores psicossociais associaram-se com a freqüência de escovação, uso diário de fio dental e motivo preventivo da visita aos serviços odontológicos. Porém, no que se refere à freqüência de utilização desses serviços, não se observou associação, talvez pelo fato de que a decisão de consultar o serviço seja dos pais e não do próprio jovem. O hábito de escovação e uso de fio dental dependem mais de fatores internos como autoestima e autocuidado, enquanto que a utilização de serviços está mais relacionada a fatores externos. Em relação ao estilo de vida, somente o consumo de açúcar esteve associado à busca de atendimento curativo.

Acredita-se que o conhecimento da situação de saúde bucal de uma população e dos fatores que podem influenciá-la é essencial para promover mudanças de comportamento que levem a atitudes positivas em relação aos cuidados com a mesma. A garantia de acesso e a qualificação contínua dos serviços de saúde bucal são imprescindíveis na busca de melhores condições de vida e saúde para nossos jovens.

Nesse contexto, a escola deve ser um ambiente favorável à saúde, tendo o papel de fornecer informações e conhecimentos que habilitem o jovem a lidar melhor com a adversidade, contribuindo para que se torne mais consciente de suas escolhas e menos vulnerável aos fatores de risco 26 . Por meio de ações que fortaleçam a auto-estima e as relações sociais e familiares, pode capacitar seus alunos para opções de vida mais saudáveis. Ressalta-se a necessidade de realização de estudos sobre o tema, por sua relevância para definição de estratégias de promoção da saúde que visem enfrentar as causas dos problemas, não apenas as suas conseqüências. 


\section{Resumo}

Estudo transversal com 1.170 adolescentes da sétima série da rede pública municipal de Gravataí, Rio Grande do Sul, Brasil, investigando a associação de fatores sócio-demográficos, psicossociais e relacionados ao estilo de vida com hábitos de saúde bucal e utilização de serviços odontológicos. Os dados foram analisados por regressão de Cox modificada para estudos transversais. As meninas tiveram maior freqüência de escovação dentária assim como aqueles que relataram não se sentirem discriminados ou sozinhos. O uso diário de fio dental associou-se à melhor inserção sócio-econômica, à busca por serviços privados, à compreensão dos pais e à ausência de sentimento de solidão. A freqüência anual de utilização de serviços odontológicos foi maior entre os de inserção sócio-econômica mais alta. Houve maior busca de serviços por motivo preventivo entre aqueles com melhor inserção sócio-econômica, os que se sentiram compreendidos pelos pais e os que não costumavam consumir doces. Os hábitos de saúde bucal apresentaram associação com inserção sócio-econômica familiar e com fatores psicossociais, exceção à utilização anual de serviços. Quanto ao estilo de vida, o baixo consumo de doces repercutiu positivamente sobre o motivo da visita ao serviço.

Serviços de Saúde Bucal; Adolescente; Saúde Bucal

\section{Colaboradores}

R. S. Davoglio participou desde a coleta e análise dos dados até a redação final do artigo. D. R. G. C. Aerts colaborou em todas as etapas do estudo, desde o planejamento do mesmo até a análise dos dados e no processo de redação do artigo. C. Abegg contribuiu no planejamento do estudo e no processo de redação do artigo. S. L. Freddo participou na coleta dos dados e redação da versão final do artigo. L. Monteiro colaborou no planejamento do estudo, coleta dos dados e redação da versão final do artigo.

\section{Referências}

1. Freeman R. The determinants of dental health attitudes and behaviours. Br Dent J 1999; 187:15-8.

2. World Health Organization. Nutrition in adolescence: issues and challenges for the health sector: issues in adolescent health and development. Geneva: World Health Organization; 2005. (WHO Discussion Papers on Adolescence).

3. Valente MSG. Adolescencia y salud bucal. Adolesc Latinoam 1998; 1:170-4.

4. Matos DL, Lima-Costa MFF, Guerra HL, Marcenes W. Projeto Bambuí: estudo de base populacional dos fatores associados com o uso regular de serviços odontológicos em adultos. Cad Saúde Pública 2001; 17:661-8.

5. Matos DL, Lima-Costa MFF, Guerra HL, Marcenes W. Projeto Bambuí: avaliação de serviços odontológicos privados, públicos e de sindicatos. Rev Saúde Pública 2002; 36:237-43.
6. Abegg C. Desenvolvimento de comportamentos e hábitos condutores à saúde bucal. In: Bönecker $\mathrm{M}$, Sheiham A, organizadores. Promovendo a saúde bucal na infância e adolescência: conhecimentos e práticas. Porto Alegre: Editora Santos; 2004. p. 97-108.

7. Avlund K, Hola-Petersen P, Morse DE, Viitanen M, Winblad B. Social relations as determinants of oral health among persons over the age of 80 years. Community Dent Oral Epidemiol 2003; 31:454-62.

8. Rickardsson B, Hanson BS. Social network and regular dental care utilisation in elderly men. Results from the population study "Men born in 1914", Malmö, Sweden. Swed Dent J 1989; 13:151-61.

9. Andrade GRB, Vaitsman J. Apoio social e redes: conectando solidariedade e saúde. Ciênc Saúde Coletiva 2002; 7:925-34. 
10. Ylöstalo PV, Ek E, Laitinen J, Knuuttila ML. Optimism and life satisfaction as determinants for dental and general health behavior oral health habits linked to cardiovascular risk factors. J Dent Res 2003; 82:194-9.

11. Shou L, Curry C, McQueen D. Using a "life-style" perspective to understand toothbrushing behavoir in Scottish schoolchildren. Community Dent Oral Epidemiol 1990; 18:230-4.

12. Wickholm S, Galanti MR, Söder V, Gilljam H. Cigarette smoking, snuff use and alcohol drinking: coexisting risk behaviours for oral health in young males. Community Dent Oral Epidemiol 2003; 31:269-74.

13. Pilinová A, Krutina M, Salandová M, Pilin A. Oral health status of drug addicts in the Czech Republic. J Forensic Odontostomatol 2003; 21:36-9.

14. Ruzany MH, Szwarcwald CL. Oportunidades perdidas de atenção integral ao adolescente: resultado de estudo piloto. Adolesc Latinoam 2000; 2:26-35.

15. World Health Organization. Global school-based student health survey (GSHS). http://www.who. $\mathrm{int/chp/gshs/en/} \mathrm{(acessado} \mathrm{em} \mathrm{12/Dez/2007).}$

16. Coordenação Geral de Doenças e Agravos não Transmissíveis, Departamento de Análise de Situação de Saúde, Secretaria de Vigilância em Saúde, Ministério da Saúde. Monitoramento da saúde do adolescente. http://portal.saude.gov.br/portal/ arquivos/pdf/projeto_escolares.pdf (acessado em 12/Dez/2007).

17. Departamento de Atenção Básica, Secretaria de Atenção à Saúde, Ministério da Saúde. Cadernos de Atenção Básica nº. 17: saúde bucal. Brasília: Ministério da Saúde; 2006. (Série A. Normas e Manuais Técnicos).

18. Pinheiro RS, Aguiar FP, Sass PE, Vilela MJN. Diferenças no uso de serviços odontológicos entre os estados do Brasil: uma análise baseada em modelos hierárquicos. Cad Saúde Colet (Rio J) 2006; 14:141-8.

19. Associação Brasileira de Empresas de Pesquisa. Critério de classificação econômica Brasil. http:// www.abep.org/codigosguias/ABEP_CCEB.pdf (acessado em 12/Dez/2007).

20. Victora CG, Huttly S, Fuchs S, Olinto MT. The role of conceptual frameworks in epidemiological analysis: a hierarchical approach. Int J Epidemiol 1997; 26:224-7.

21. Abegg C. Hábitos de higiene bucal dos adultos porto-alegrenses. Rev Saúde Pública 1997; 31: 586-93.
22. Lisbôa IC, Abegg C. Hábitos de higiene bucal e uso de serviços odontológicos por adolescentes e adultos do Município de Canoas, Estado do Rio Grande do Sul, Brasil. Epidemiol Serv Saúde 2006; 15:29-39.

23. Ferriani MGC, Dias TS, Silva KZ, Martins CS. Auto-imagem corporal de adolescentes atendidos em um programa multidisciplinar de assistência ao adolescente obeso. Rev Bras Saúde Matern Infant 2005; 5:27-33.

24. Sanders AE, Spencer AJ, Slade GD. Evaluating the role of dental behaviour in oral health inequalities. Community Dent Oral Epidemiol 2006; 34:71-9.

25. Ruzany MH. Mapa da situação de saúde do adolescente no Município do Rio de Janeiro [Tese de Doutorado]. Rio de Janeiro: Escola Nacional de Saúde Pública, Fundação Oswaldo Cruz; 2000.

26. Secretaria de Políticas de Saúde, Ministério da Saúde. A promoção da saúde no contexto escolar. Rev Saúde Pública 2002; 36:533-5.

27. Dolic M, Bailer J, Staehle HJ, Eickholz P. Psychosocial factors as risk indicators of periodontitis. J Clin Periodontol 2005; 32:1134-40.

28. Anttila S, Knuuttila M, Ylöstalo P, Joukamaa M. Symptoms of depression and anxiety in relation to dental health behavior and self-perceived dental treatment need. J Oral Sci 2006; 14:109-14.

29. Sawyer DO, Leite IC, Alexandrino R. Perfis de utilização de serviços de saúde no Brasil. Ciênc Saúde Coletiva 2002; 7:757-76.

30. Barros AJD, Bertoldi AD. Desigualdades na utilização e no acesso a serviços odontológicos: uma avaliação em nível nacional. Ciênc Saúde Coletiva 2002; 4:709-17.

31. Watt R, Sheiham A. Inequalities in oral health: a review of the evidence and recommendations for action. Br Dent J 1999; 187:6-12.

32. Moreira TP, Nations MK, Alves SCF. Dentes da desigualdade: marcas bucais da experiência vivida na pobreza pela comunidade do Dendê, Fortaleza, Ceará, Brasil. Cad Saúde Pública 2007; 23:1383-92.

33. Maltz M, Silva BB. Relação entre cárie, gengivite e fluorose e nível socioeconômico em escolares. Rev Saúde Pública 2001; 35:170-6.

34. Peres KGA, Bastos JRM, Latorre MRDO. Severidade de cárie em crianças e relação com aspectos sociais e comportamentais. Rev Saúde Pública 2000; 34:402-8.

Recebido em 12/Mar/2008

Versão final reapresentada em 04/Set/2008 Aprovado em 01/Out/2008 